\title{
Cremmer-Gervais Quantum Lie Algebra
}

\author{
Oleg Ogievetsky* \\ Centre de Physique Théorique, Luminy, 13288 Marseille, France
}

Todor Popov

Institute for Nuclear Research and Nuclear Energy, Bulgarian Academy of Sciences, Sofia, BG-1784, Bulgaria

\begin{abstract}
We describe a quantum Lie algebra based on the Cremmer-Gervais R-matrix. The algebra arises upon a restriction of an infinite-dimensional quantum Lie algebra.
\end{abstract}

\section{Introduction}

The notion of a quantum Lie algebra is a modification of the notion of a Lie algebra. Quantum Lie algebras arise as the algebras generated by the quantum analogs of vector fields in the framework of the bicovariant differential calculus on quantum groups [1] (for an introduction see e.g. [2]). Many constructions from the theory of Lie algebras can be generalized for quantum Lie algebras (for example, the standard complex, BRST operator etc. [3, 4, 5]).

${ }^{*}$ On leave of absence from P.N. Lebedev Physical Institute, Theoretical Department, Leninsky prospekt 53, 119991 Moscow, Russia

†Unité Mixte de Recherche (UMR 6207) du CNRS et des Universités Aix-Marseille I, AixMarseille II et du Sud Toulon - Var; laboratoire affilié à la FRUMAM (FR 2291) 
In this Note we outline the quantum Lie algebra having the so called CremmerGervais R-matrix [7] as the braid matrix. This can be seen as a first step in constructing the BRST operator for the bicovariant differential calculus based on the Cremmer-Gervais R-matrix.

\section{Quantum Lie Algebra}

The bicovariant differential calculus is characterized by functionals $\chi_{i}$ and $f_{j}^{i}$ on a Hopf algebra $\mathcal{A}$ ("the algebra of functions on a quantum group") satisfying the relations

$$
\begin{array}{ll}
\chi_{i} \chi_{j}-\sigma_{i j}^{k l} \chi_{k} \chi_{l}=C_{i j}^{k} \chi_{k}, & \sigma_{i j}^{k l} f_{k}^{a} f_{l}^{b}=f_{i}^{k} f_{j}^{l} \sigma_{k l}^{a b}, \\
\sigma_{i j}^{k l} \chi_{k} f_{l}^{a}+C_{i j}^{l} f_{l}^{a}=f_{i}^{k} f_{j}^{l} C_{k l}^{a}+f_{i}^{a} \chi_{j}, & \chi_{i} f_{j}^{a}=\sigma_{i j}^{k l} f_{k}^{a} \chi_{l} .
\end{array}
$$

Here the structure constants $C_{j k}^{i}$ and the braid matrix $\sigma_{k l}^{i j}\left(C_{j k}^{i}\right.$ and $\sigma_{k l}^{i j}$ are subject to certain conditions, see below) are such that $C_{j k}^{i}=\chi_{k}\left(M_{j}^{i}\right)$ and $\sigma_{k l}^{i j}=f_{l}^{i}\left(M_{k}^{j}\right)$, where the matrix $M \in \mathcal{A}$ is given by the right coaction on the space of leftinvariant forms

$$
\Delta_{R}\left(\omega^{i}\right)=\omega^{j} \otimes M_{j}^{i}, \quad M_{j}^{i} \in \mathcal{A}, \quad \omega^{i} \in \Gamma .
$$

The algebra (1) endowed with the comultiplication $\Delta$, counit $\epsilon$ and antipode $S$,

$$
\begin{array}{lll}
\Delta f_{j}^{i}=f_{k}^{i} \otimes f_{j}^{k}, & \epsilon\left(f_{j}^{i}\right)=\delta_{j}^{i}, & S\left(f_{k}^{i}\right) f_{j}^{k}=\delta_{j}^{i}=f_{k}^{i} S\left(f_{j}^{k}\right), \\
\Delta \chi_{i}=1 \otimes \chi_{i}+\chi_{j} \otimes f_{i}^{j}, & \epsilon\left(\chi_{i}\right)=0, & S\left(\chi_{i}\right)=-\chi_{j} S\left(f_{i}^{j}\right),
\end{array}
$$

becomes a Hopf algebra which we will be denote by $\mathcal{L}$. The subalgebra generated by $\chi_{i}$ is called quantum Lie algebra.

The relations for $\mathcal{L}$ can be written in a concise way with the help of a single $\mathrm{R}$-matrix [6]. Let us make a convention that the small indices $i, j, \ldots, k$ run over a set $\mathcal{I}$ and the capital indices $I, J, \ldots, K$ run over the set $\mathcal{I}_{0}:=0 \cup \mathcal{I}$. Denote by $\hat{R}$ and $T$ the following matrices

$$
\hat{R}_{k L}^{I j}=\left(\begin{array}{cc}
\delta_{k}^{j} & C_{k l}^{j} \\
0 & \sigma_{k l}^{i j}
\end{array}\right), \quad \hat{R}_{0 L}^{I 0}=\delta_{L}^{I}, \quad T_{J}^{I}=\left(\begin{array}{cc}
1 & \chi_{j} \\
0 & f_{j}^{i}
\end{array}\right),
$$

i.e., $\hat{R}_{k l}^{i j}=\sigma_{k l}^{i j}, \hat{R}_{k l}^{0 j}=C_{k l}^{j}, \hat{R}_{B 0}^{0 A}=\delta_{B}^{A}, \hat{R}_{0 B}^{A 0}=\delta_{B}^{A}$ and $T_{j}^{i}=f_{j}^{i}, T_{j}^{0}=\chi_{j}, T_{0}^{0}=1$ and all others entries are equal to zero. Suppose now that $R$ is a solution of the Yang-Baxter equation

$$
\hat{R}_{12} \hat{R}_{23} \hat{R}_{12}=\hat{R}_{23} \hat{R}_{12} \hat{R}_{23}
$$


Then the Hopf algebra relations (1) and (2) are equivalent to

$$
\begin{array}{ll}
\hat{R}_{I J}^{K L} T_{K}^{A} T_{L}^{B}=T_{I}^{K} T_{J}^{L} \hat{R}_{K L}^{A B}, & \Delta T_{J}^{I}=T_{K}^{I} \otimes T_{J}^{K}, \\
S\left(T_{K}^{I}\right) T_{J}^{K}=\delta_{J}^{I}=T_{K}^{I} S\left(T_{J}^{K}\right), & \epsilon\left(T_{J}^{I}\right)=\delta_{J}^{I} .
\end{array}
$$

The Yang-Baxter relation for $\hat{R}$ implies, for the components $\sigma_{k l}^{i j}$ and $C_{k l}^{j}$,

$$
\begin{array}{ll}
C_{n i}^{s} C_{s j}^{m}-\sigma_{i j}^{k l} C_{n k}^{s} C_{s l}^{m}=C_{i j}^{k} C_{n k}^{m}, & \sigma_{i j}^{k l} \sigma_{n k}^{a s} \sigma_{s l}^{b m}=\sigma_{n i}^{k s} \sigma_{s j}^{l m} \sigma_{k l}^{a b}, \\
\sigma_{i j}^{k l} C_{n k}^{s} \sigma_{s l}^{a m}+C_{i j}^{l} \sigma_{n l}^{a m}=\sigma_{n i}^{k s} \sigma_{s j}^{l m} C_{k l}^{a}+\sigma_{n i}^{a s} C_{s j}^{m}, & C_{n i}^{s} \sigma_{s j}^{a m}=\sigma_{i j}^{k l} \sigma_{n k}^{a s} C_{s l}^{m} .
\end{array}
$$

Here the first relation is the "braided" Jacobi identity and the second one is simply the braid relation for $\sigma, \sigma_{23} \sigma_{12} \sigma_{23}=\sigma_{12} \sigma_{23} \sigma_{12}$.

Given a braid matrix $\sigma$ it is natural to ask if non-zero structure constants $C_{i j}^{k}$ consistent with $\sigma$ exist or is there a non-trivial quantum Lie algebra structure compatible with $\sigma$. As we have seen this question is equivalent to finding a suitable extension (3) of the R-matrix $\sigma$.

In this Note we obtain an infinite-dimensional R-matrix which upon restrictions yields finite-dimensional quantum Lie algebras compatible with the Cremmer-Gervais $R$-matrix [7].

\section{Cremmer-Gervais extended}

We apply the elegant method used in [8] and then in [9] where the Yang-Baxter operators are realized as operators in a certain space of functions. Finite-dimensional $\mathrm{R}$-matrices arise upon a restriction of the operator domain to an appropriate invariant finite-dimensional subspace, such as the space of polynomials of bounded degree.

For a ring $K$, let $K(x)$ be the ring of rational functions in $x$ with coefficients in $K$. An endomorphism of $K$ extends to an endomorphism of $K(x)$ (which acts only on the coefficients of a rational function). Having an endomorphism $\phi \in$ End $\mathbb{C}(x, y)$, introduce $\phi_{12} \in$ End $\mathbb{C}(x, y, z)$ considering $\mathbb{C}(x, y, z)$ as $\mathbb{C}(x, y)(z)$. In the same vein, $\phi_{13} \in$ End $\mathbb{C}(x, z)(y)$ and $\phi_{23} \in \operatorname{End} \mathbb{C}(y, z)(x)$ and the functional Yang-Baxter equation reads $\phi_{12} \phi_{13} \phi_{23}=\phi_{23} \phi_{13} \phi_{12}$.

Given a rational function $F(x, y)$ with series expansion (around 0 ) $F(x, y)=$ $\sum_{i, j \in \mathbb{Z}} F_{i, j} x^{i} y^{j}$, define the operation $\operatorname{reg}_{x, y}$ which maps $F(x, y)$ to the non-singu- 
lar part $f(x, y)$ of its expansion,

$$
f(x, y)=\operatorname{reg}_{x, y} F(x, y):=\sum_{i, j \geq 0} F_{i, j} x^{i} y^{j} .
$$

Theorem 1. Let $\hat{R}$ be the following linear operator in $\operatorname{End} \mathbb{C}(x, y)$

$$
\hat{R}=P+\beta \frac{y}{x-y}(P-I) r e g_{x, y}+\frac{C}{x} \operatorname{eval}_{x=0}(P-I) r e g_{x, y},
$$

$\beta=1-q^{-2}$ and $C$ are arbitrary constants. Here I stands for the identity operator, $P$ for the permutation $(P F)(x, y)=F(y, x)$ and eval $x_{x=0}$ is the evaluation at $x=0$; in other words, for an arbitrary $F(x, y) \in \mathbb{C}(x, y)$ the result of the action of the operator $\hat{R}$ reads

$$
(\hat{R} F)(x, y)=F(y, x)+\beta y \frac{f(y, x)-f(x, y)}{x-y}+C \frac{f(y, 0)-f(0, y)}{x} .
$$

The operator $\hat{R}$ satisfies the braid equation $\hat{R}_{12} \hat{R}_{23} \hat{R}_{12}=\hat{R}_{23} \hat{R}_{12} \hat{R}_{23}$.

Proof. The braid equation for the operator $\hat{R}$ is equivalent to the Yang-Baxter equation $R_{12} R_{13} R_{23}=R_{23} R_{13} R_{12}$ for $R=P \hat{R}$. The operator $r$ defined through $R=I+r$ reads

$$
r=\beta \frac{x}{x-y}(P-I) \operatorname{reg}_{x, y}+\frac{C}{y} \operatorname{eval}_{y=0}(I-P) r e g_{x, y} .
$$

It acts on a function $F(x, y)$ as follows: $(r F)(x, y)=\beta(\rho F)(x, y)+C(\mathfrak{s} F)(x, y)$, where

$$
(\rho F)(x, y)=x \frac{f(y, x)-f(x, y)}{x-y}, \quad(\mathfrak{s} F)(x, y)=\frac{f(x, 0)-f(0, x)}{y} .
$$

Note that $r$ sends a polynomial $f(x, y)$ to the sum of a polynomial in $x$ and $y$ (the term with the coefficient $\beta$ ) and a polynomial in $x$ (with the coefficient $\frac{C}{y}$ ).

The first step is to check that $r$ is a classical r-matrix.

Lemma 2. The operator $r$ satisfies the classical Yang-Baxter equation

$$
\left[r_{12}, r_{13}\right]+\left[r_{12}, r_{23}\right]+\left[r_{13}, r_{23}\right]=0 \text {. }
$$


Proof of the lemma. The operator in the left hand side of (8) depends only on the regular part of a function $F(x, y, z) \in \mathbb{C}(x, y, z)$ therefore it is enough to check the assertion on an arbitrary polynomial $f(x, y, z) \in \mathbb{C}[x, y, z]$. Since the coefficients $\beta$ and $C$ are arbitrary, the classical Yang-Baxter equation for $r$ splits into three components. The component proportional to $\beta^{2}$ is the classical YangBaxter equation for $\rho$; it is satisfied: $\rho$ is the classical Cremmer-Gervais $r$-matrix [9, 10]. Next, a straightforward verification shows that

$$
\begin{gathered}
\rho_{13} \mathfrak{s}_{23}=0, \rho_{23} \mathfrak{s}_{13}=0, \rho_{23} \mathfrak{s}_{12}=0,\left[\mathfrak{s}_{12}, \rho_{13}\right]+\mathfrak{s}_{12} \rho_{23}=0, \\
{\left[\rho_{12}, \mathfrak{s}_{13}\right]+\mathfrak{s}_{13} \rho_{23}-\mathfrak{s}_{23} \rho_{13}+\left[\rho_{12}, \mathfrak{s}_{23}\right]=0}
\end{gathered}
$$

on polynomials. The sum (with corresponding signs) of these equalities is the component proportional to $\beta C$. Finally, a straightforward verification shows that

$$
\mathfrak{s}_{23} \mathfrak{s}_{12}=0, \mathfrak{s}_{23} \mathfrak{s}_{13}=0, \mathfrak{s}_{13} \mathfrak{s}_{23}=0,\left[\mathfrak{s}_{12}, \mathfrak{s}_{13}\right]+\mathfrak{s}_{12} \mathfrak{s}_{23}=0
$$

on polynomials and the classical Yang-Baxter equation for the operator $\mathfrak{s}$ (the component, proportional to $C^{2}$ ) follows.

The Yang-Baxter equation for $R=I+r$ holds true if the operator $r$ satisfies the classical Yang-Baxter equation and the Yang-Baxter equation

$$
r_{12} r_{13} r_{23}=r_{23} r_{13} r_{12} .
$$

To check this identity on an arbitrary function $F(x, y, z) \in \mathbb{C}(x, y, z)$ it is again enough to check it on an arbitrary polynomial function $f(x, y, z) \in \mathbb{C}[x, y, z]$. Now (9) splits into four components. The component proportional to $\beta^{3}$ vanishes ( $\rho$ satisfies a stronger equation, see [10]). Next, a direct verification shows that

$$
\begin{gathered}
\mathfrak{s}_{12} \rho_{13} \rho_{23}=0, \quad \rho_{12} \rho_{13} \mathfrak{s}_{23}=0, \quad \rho_{23} \mathfrak{s}_{13} \rho_{12}=0, \\
\rho_{23} \rho_{13} \mathfrak{s}_{12}=0, \quad \rho_{12} \mathfrak{s}_{13} \rho_{23}=\mathfrak{s}_{23} \rho_{13} \rho_{12}
\end{gathered}
$$

on polynomials; the vanishing of the component proportional to $\beta^{2} C$ follows. Finally, each term in the components, proportional to $\beta C^{2}$,

$$
\begin{aligned}
& \mathfrak{s}_{12} \mathfrak{s}_{13} \rho_{23}=0, \quad \mathfrak{s}_{12} \rho_{13} \mathfrak{s}_{23}=0, \quad \rho_{12} \mathfrak{s}_{13} \mathfrak{s}_{23}=0, \\
& \rho_{23} \mathfrak{s}_{13} \mathfrak{s}_{12}=0, \quad \mathfrak{s}_{23} \rho_{13} \mathfrak{I}_{12}=0, \quad \mathfrak{s}_{23} \mathfrak{s}_{13} \rho_{12}=0,
\end{aligned}
$$


and $C^{3}$,

$$
\mathfrak{s}_{12} \mathfrak{s}_{13} \mathfrak{s}_{23}=0, \mathfrak{s}_{23} \mathfrak{s}_{13} \mathfrak{s}_{12}=0
$$

vanishes separately, which ends the proof of the theorem.

Let $V=\bigoplus_{i=0}^{n} \mathbb{C} e_{i}$ be a finite-dimensional vector space of functions $\frac{p(x)}{x}$ where $p(x)$ is a polynomial of degree not higher than $n$. Identify $V \otimes V$ with the space of functions $\frac{p(x, y)}{x y}$ where $p(x, y)$ is a polynomial of degree not higher than $n$ in $x$ and not higher than $n$ in $y$. The space $V \otimes V$ is stable under the action of the operator $\hat{R}$. The matrix of the restricted operator $\hat{R}\left(e_{K} \otimes e_{L}\right)=$ $\sum_{I, J=0}^{n} e_{I} \otimes e_{J} \hat{R}_{K L}^{I J}$ (which we denote again by $\hat{R}$ ) is given by

$$
\hat{R}\left(x^{K-1} y^{L-1}\right)=\sum_{I, J=0}^{n} \hat{R}_{K L}^{I J} x^{I-1} y^{J-1} ; \quad I, J, K, L=0, \ldots, n .
$$

The non-vanishing entries of the matrix $\hat{R}_{K L}^{I J}$ read as follows

$$
\begin{gathered}
\hat{R}_{K 0}^{0 J}=\hat{R}_{0 K}^{J 0}=\delta_{K}^{J}, \quad \hat{R}_{k l}^{0 j}=C_{k l}^{j}=C\left(\delta_{l}^{1} \delta_{k}^{j}-\delta_{k}^{1} \delta_{l}^{j}\right), \\
\hat{R}_{k l}^{i j}=\delta_{l}^{i} \delta_{k}^{j}+\left(1-q^{-2}\right)\left(\sum_{k \leq s<l}-\sum_{l \leq s<k}\right) \delta_{s}^{i} \delta_{k+l-s}^{j},
\end{gathered}
$$

$i, j, k, l=1, \ldots, n$.

The latter submatrix $\hat{R}_{k l}^{i j}$ is the member (with $p=1$ ) of the Cremmer-Gervais family of non-unitary R-matrices

$$
\left(\hat{R}_{C G, p}\right)_{k l}^{i j}=p^{k-l} \delta_{l}^{i} \delta_{k}^{j}+\left(1-q^{-2}\right)\left(\sum_{k \leq s<l}-\sum_{l \leq s<k}\right) p^{k-s} \delta_{s}^{i} \delta_{k+l-s}^{j} .
$$

We sum up these results in the following corollary.

Corollary 3. The above finite-dimensional restriction of the operator $\hat{R}$, defined by (5), gives rise to a quantum Lie algebra associated with the $p=1$ member of the family of non-unitary Cremmer-Gervais R-matrices. The non-zero structure constants $C_{k l}^{j}$ are all equal to $\pm C$ (the constant $C$ can be set to 1 by rescalings),

$$
C_{j 1}^{j}=-C_{1 j}^{j}=C, \quad j=1 \ldots n .
$$


Remark 1. Our treatment is an extension of the construction of [9] in which the finite-dimensional Cremmer-Gervais matrices arise upon restrictions of infinitedimensional functional R-matrices to the spaces of polynomials. The boundary (unitary) Cremmer-Gervais solution of the Yang-Baxter equation can be treated along the same lines [9]. The boundary Cremmer-Gervais R-matrix as well gives rise to a quantum Lie algebra which will be described elsewhere.

\section{Acknowledgements}

The work was partially supported by the ANR project GIMP No.ANR-05-BLAN0029-01. T.P. thanks Centre de Physique Théorique, Luminy for the hospitality. The work of T.P. was also partially supported by the European Operational program HRD through contract BGO051PO001/07/3.3-02/53 with the Bulgarian Ministry of Education.

\section{References}

[1] S. L. Woronowicz, Comm. Math. Phys. 122 (1989) 125-170.

[2] P. Aschieri and L. Castellani, Int. J. Mod. Phys. A 8 (1993) 1667.

[3] A. P. Isaev and O. V. Ogievetsky, Theoretical and Math. Physics 129 (2001) 1558-1572.

[4] A. P. Isaev and O. V. Ogievetsky, Int. J. Math. Phys. A 19 (2004) 240-247.

[5] V. G. Gorbounov, A. P. Isaev and O. V. Ogievetsky, Theoretical and Math. Physics 139 (2004) 473-485.

[6] D. Bernard, Phys. Lett. B260 (1991) 389-393.

[7] E. Cremmer and J.-L. Gervais, Comm. Math. Phys. 134 (1990) 619-632.

[8] Y. Shibukawa and K. Ueno, Lett. Math. Phys. 25 (1992) 239-248.

[9] R. Endelman and T. Hodges, Lett. Math. Phys. 52 (2000) 225-237.

[10] O. Ogievetsky and T. Popov, R-matrices in Rime; ArXiv: 0704.1947 [math.QA] 